\title{
Changes in pituitary gene expression may underlie multiple domesticated traits in chickens
}

\author{
Amir Fallahshahroudi ${ }^{1} \cdot$ Pia Løtvedt ${ }^{1} \cdot$ Johan Bélteky $^{1} \cdot$ Jordi Altimiras $^{1} \cdot$ Per Jensen $^{1}$ \\ Received: 9 December 2017 / Revised: 5 April 2018 / Accepted: 26 April 2018 / Published online: 22 May 2018 \\ (c) The Genetics Society 2018
}

\begin{abstract}
Domesticated animals share a unique set of morphological and behavioral traits, jointly referred to as the domesticated phenotype. Striking similarities amongst a range of unrelated domesticated species suggest that similar regulatory mechanisms may underlie the domesticated phenotype. These include color pattern, growth, reproduction, development and stress response. Although previous studies have focused on the brain to find mechanisms underlying domestication, the potential role of the pituitary gland as a target of domestication is highly overlooked. Here, we study gene expression in the pituitary gland of the domesticated White Leghorn chicken and its wild ancestor, the Red Junglefowl. By overlapping differentially expressed genes with a previously published list of functionally important genes in the pituitary gland, we narrowed down to 34 genes. Amongst them, expression levels of genes with inhibitory function on pigmentation (ASIP), main stimulators of metabolism and sexual maturity (TSHB and DIO2), and a potential inhibitor of broodiness (PRLR), were higher in the domesticated breed. Additionally, expression of 2 key inhibitors of the stress response (NR3C1,CRHR2) was higher in the domesticated breed. We suggest that changes in the transcription of important modulatory genes in the pituitary gland can account not only for domestication of the stress response in domestic chickens, but also for changes in pigmentation, development, and reproduction. Given the pivotal role of the pituitary gland in the regulation of multiple shared domesticated traits, we suggest that similar changes in pituitary transcriptome may contribute to the domesticated phenotype in other species as well.
\end{abstract}

\section{Introduction}

A number of animal species have undergone domestication, and thereby adapted to living in captivity and in proximity to humans (Price 1999). The domesticated phenotype is a collection of similar traits evolving in many unrelated species, encompassing changes to morphology, physiology and behavior (Price 1999; Jensen 2014). These traits include, e.g., changes in color pattern, increased docility, higher growth rate, earlier sexual maturity, lack of strict seasonal

These authors contributed equally: Amir Fallahshahroudi, Pia Løtvedt.

Electronic supplementary material The online version of this article (https://doi.org/10.1038/s41437-018-0092-z) contains supplementary material, which is available to authorized users.

Per Jensen

perje@ifm.liu.se

1 AVIAN Behavioural Genomics and Physiology Group, IFM Biology, Linköping University, 58183 Linköping, Sweden reproduction, alteration of craniofacial morphology, and lowered stress response (Künzl and Sachser 1999; Price 1999).

The domesticated chicken originated from the Red Junglefowl (RJF) around 8000 years ago in Asia (Tixier-Boichard et al. 2011). Today, the chicken is the most numerous domesticated species on earth, and hundreds of diverse breeds have evolved as a result of selection for meat production, egg laying, research, and hobby (Mirkena et al. 2010). In comparison with the ancestral Red Junglefowl, domesticated chickens reach sexual maturity earlier, grow faster, lay more and bigger eggs throughout the year, are less fearful, and have an attenuated stress response (Schütz et al. 2002; Kerje et al. 2003; Ericsson et al. 2014; Fallahsharoudi et al. 2015).

The genetic basis for the majority of the modified traits during animal domestication is unknown (Wiener and Wilkinson 2011). Genetic changes causing differences in gene expression have been suggested to underlie most phenotypic differences between closely related species (Albert and Kruglyak 2015). Gene expression differences 
between domesticated and wild animals could therefore underlie the traits that are associated with domestication (Albert et al. 2012). Given the importance of the brain in regulation of physiology and behavior, several studies have focused on the effects of domestication on brain gene expression (Saetre et al. 2004; Tymchuk et al. 2009; Albert et al. 2012; Nätt et al. 2012; Heyne et al. 2014). However, other tissues, with as much or even more relevance to behavior and physiology have seldom been studied. For instance, the pituitary, a key structure in the hypothalamicpituitary-adrenal (HPA) axis as well as in the hypothalamicpituitary-gonadal (HPG) axis, has so far largely been overlooked, and we therefore decided to focus this experiment on the pituitary gland, using chickens as a suitable model species.

The pituitary gland is a neuroendocrine organ that plays a fundamental role in the regulation of multiple vital biological processes, including pathways affecting several domesticated traits (Ferris 2006; Scanes 2015). Inhibitory and stimulatory peptides with hypothalamic origin regulate the production and release of pituitary hormones. The pituitary consists of two distinct structures (anterior and posterior) that have different embryonic origins. The anterior pituitary originates from Rathke's pouch and consists of cells that produce adrenocorticotrophic hormone (ACTH), luteinizing hormone (LH), follicle stimulating hormone (FSH), prolactin, growth hormone $(\mathrm{GH})$, thyroid stimulating hormone (TSH), and the melanocyte-stimulating hormones (MSH). The posterior adrenal gland originates from the infundibulum and serves as the site for secretion of oxytocin and vasopressin (and the homolog vasotocin in birds) into the blood. The pituitary hormones can either have direct effects on other tissues (i.e., GH), or modulate activity of other endocrine tissues (i.e., adrenal and thyroid glands) (Scanes 2015).

One major trait shared by many domesticates is an attenuated stress response (Weiler et al. 1998; Künzl and Sachser 1999; Gulevich et al. 2004; Trut et al. 2009; Plyusnina et al. 2011; Ericsson et al. 2014). During stress, the hypothalamic-pituitary-adrenal axis is activated. Neural signals reach the hypothalamus, which secretes corticotropin-releasing hormone (CRH) and arginine vasopressin (AVP) into the portal vessel. These hypothalamic peptides reach their designated receptors in the anterior pituitary and stimulate the release of adrenocorticotropic hormone $(\mathrm{ACTH})$ into the general circulation. ACTH in turn is transported to the adrenal glands, and stimulates release of stress mediators such as glucocorticoids (Joëls and Baram 2009; Herman et al. 2016).

The activity of the HPA axis is mainly regulated by the negative feedback loop through the action of corticosteroids at glucocorticoid receptors (GR) and mineralocorticoid receptors (MR) that are located in the brain and pituitary gland (Keller-Wood 2015). Given its central role in regulating many biological processes, we hypothesize that gene expression changes at the level of the pituitary gland may influence multiple domesticated traits. We therefore used microarray analysis of pituitaries of ancestral Red Junglefowl and domesticated White Leghorns to obtain a broad picture of gene expression differences.

\section{Materials and methods}

\section{Ethical statement}

All experimental protocols were approved by Linköping Council for Ethical Licensing of Animal Experiments, ethical permit no 50-13. Experiments were conducted in accordance with the approved guidelines.

\section{Animals and housing}

We studied a captive population of ancestral Red Junglefowl and one population of domesticated White leghorn (WL). The RJF population that was used in this experiment was originally brought to Sweden from wild population in Thailand to a Zoological park in northern Sweden (Frösö Zoo). Based on the previous habitat and the appearance of the acquired RJF, these birds most likely were of the subspecies G. g. gallus or G. g. spadiceus (Schütz et al. 2001). We acquired 1-day-old chickens from Frösö Zoo, kept, and bred them in our animal facility in Linköping Sweden for 16 generations with a population size of around 100 with pedigree breeding. SLU13 originates from the Scandinavian selection and crossbreeding experiment (Liljedahl et al. 1979) and was maintained at the Swedish University of Agricultural Sciences. SLU13 line developed for research purposes and selected for egg mass but does not represent any commercial strain of birds (Schütz et al. 2001). We currently have a population size of around 100 individuals per generation of SLU13 at our facility at Linköping, Sweden. For this study, we collected and incubated fertile eggs from floor-housed flocks of 30-40 females and 6 males for both populations simultaneously. The population of RJF that was used in this study has a relatively long history of living in captivity, and therefore we can speculate that factors such as genetic drift and unintentional selection might have influenced it. However, compared to the domesticated egg layer breeds, the RJF birds are smaller, show more fearful behavior, have a lower HPA axis reactivity, lay less and smaller eggs and show seasonal reproduction behavior such as broodiness (Schütz et al. 2001; Ericsson et al. 2014). Therefore, although the studied RJF population does not represent the true ancestral population, it is more like wild-living Red Junglefowl than to WL. 
However, to be able to generalize the findings of this study to other chicken breeds, more wild populations as well as other domesticated breeds selected for diverse production traits, and landrace chickens should be studied.

RJF and WL chicks were hatched, and thereafter kept under $12 \mathrm{~h}$ light and dark periods with ad libitum access to food and water in pen sized $1 \mathrm{~m} \times 2 \mathrm{~m}$. Due to the distinct phenotypic and behavioral differences between domesticated WL and RJF, when kept together in a single pen, WL and RJF form separate groups based on their breed (previous observations), and therefore, one group may systematically affect the other group, for instance, by pecking or preventing them to access food and water. Thus, we kept the breeds in separate pens divided into two mixedsex groups per breed.

\section{Tissue collection}

We chose the age of 6 weeks for this study because this is when phenotypic differences between the breeds and the sexes become obvious. A random sample of 12 animals from each breed, six of each sex, were culled and sampled under calm conditions, and an additional 12 animals from each breed, also six of each sex, were exposed to $15 \mathrm{~min}$ of stress by means of physical restraint in a net before culling (in total 48 chickens). Culling was performed by decapitation, and dissection took place immediately after. The whole brain was removed, and the pituitary was retrieved. The tissues were frozen in liquid nitrogen within ten minutes of sacrifice, and subsequently stored at $-80{ }^{\circ} \mathrm{C}$ until further processing.

\section{Gene expression analysis}

Total RNA was isolated from each individual sample using TRIzol $^{\text {TM }}$ reagent (Invitrogen, Carlsbad, CA, USA), according to the manufacturer's instructions. RNA purity and integrity were checked in a Bioanalyzer 2100 system (Agilent Technologies, Palo Alto, CA, USA). RNA integrity number (RIN) was larger than 8.0 in all samples used for microarray analysis. RNA was standardized in concentration, and samples were pooled so that each pool contained RNA from two birds. The two birds were from the same breed, sex and treatment. Since the animals had been kept divided into two mixed-sex groups per breed, we selected one bird from each group for each pool. Six samples resulted in a very poor yield and were not used for microarray analysis, thus six microarray samples could not be pooled and consisted of just one individual each. In total, we therefore had 24 microarray samples, out of which 18 were pools with RNA from two individuals, and six contained RNA from only one individual. The information regarding each sample as well as the details of pooling are provided in Supplementary Table 1.

Microarray probe sequences are originally designed based on RefSeq mRNA or Ensembl transcripts (WASHUC2.1/galGal3) as previously described (Johnsson et al. 2016). However the probe sets were later updated to Ensembl (version 85; (Yates et al. 2016)) according to previous description (Johnsson et al. 2018). Each probe set gives the average value of 2-3 probes, and in total the array included 20,771 probe sets. Amongst all included probe sets 16,360 were annotated to a genomic location and the rest were probe sequences from a chicken brain cDNA library (Boardman et al. 2002). The probe sequences were controlled for presence of known SNP in the founder populations by masking all known SNP positions based on resequencing of Red Junglefowl and domesticated chickens (Rubin et al. 2010).

Total RNA was labeled with Agilent Low Input Quick Amp Labeling Kit One Color (Agilent) and hybridized to $8 \times 60 \mathrm{~K}$ SurePrint G3 Custom Gene Expression Microarrays (Agilent). Scanning was performed with a MS200 Microarray scanner (Roche NimbleGen, USA). Array data were extracted using the Feature Extraction Software v12.0 (Agilent Technologies, USA). We chose to use microarrays over RNA sequencing in this experiment, since our lab has extensive experience with this method. Gene expression profiles generated by microarrays are highly correlated with that observed with RT-QPCR, although RNA sequencing is superior in detecting low abundance and novel transcripts. We also validated the expression of ten functionally important genes using RT-PCR. Six biological replicates for each breed, sex and treatment $(n=48)$ were used for cDNA synthesis using Maxima first strand cDNA synthesis kit for RT-qPCR with dsDNase (Thermo Fisher Scientific, USA). The reactions were performed in the Light Cycler 480 (Roche Diagnostics, Basel, Switzerland). We previously have published the details for performing the RT-qPCR (Fallahsharoudi et al. 2015; Løtvedt et al. 2017). The primer sequences for RT-qPCR can be found in Supplementary Table 2 .

\section{Data analysis}

All data analysis was performed using R (http://www.rproject.org) and Bioconductor (www.bioconductor.org). All expression data was normalized, and control probes were subsequently removed from the following analyses. Mean values from the probes for each gene were calculated and used in further analyses. The quality of the data was assessed through logarithmic box plots of overall expression signals, as well as with PCA analysis. No outlying samples were detected (Supplementary Figure 1). 


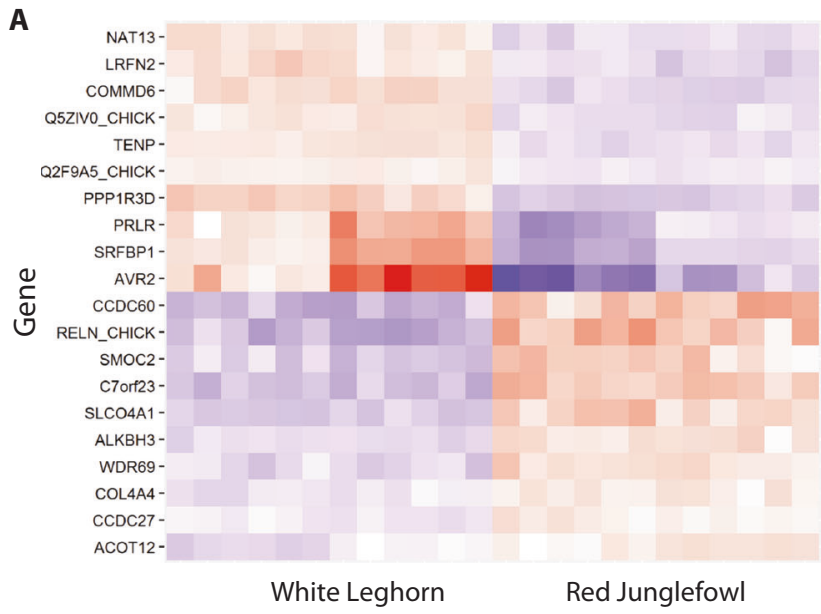

Fig. 1 Normalized gene expression profile of the pituitary gland of domesticated White Leghorn and Red Junglefowl. a The 10 top genes that are expressed higher in WL and in RJF. b Differentially expressed genes between the two breeds (FDR corrected $p$-value threshold 0.05

To find differently expressed genes, we used linear modeling with the limma package for $\mathrm{R}$ (Ritchie et al. 2015). The model used included breed, sex and treatment as fixed predictors, as well as the interaction between breed and treatment (gene expression $\sim$ breed + sex + treatment + breed $\times$ treatment). We adjusted $p$-values for multiple testing using the FDR approach (Benjamini and Hochberg 1995). We also set a threshold of 0.5 for the $\log$ fold change. For hierarchical clustering and generation of heatmaps, we used the hclust function with default parameters from the stats package in $\mathrm{R}$, in combination with the ggplot2 package (Wickham 2009). Ensembl transcript IDs extracted from all probe IDs that were differentially expressed between breed, sex and treatment groups were used for gene ontology analysis through the Database for Annotation, Visualization and Integrated Discovery (DAVID version 6.8, Oct. 2016) (Huang et al. 2008). Next, we overlapped the list of the annotated genes that were differentially expressed (DE) between the breeds with a published list containing 407 "pituitary-important" genes in chickens (Pritchett et al. 2017). This list was based on RNA sequencing of multiple tissues (pituitary, abdominal fat pad, heart fat pad, breast muscle, cerebellum, heart, liver, duodenum, jejunum, ileum, spleen, retina, pineal and hypothalamus) in chickens. These genes were either exclusively expressed in the chicken pituitary gland at day 42 or were more abundantly expressed in the pituitary compared to the other tissues at this age (Pritchett et al. 2017). We also overlapped the breed DE genes with the list of 265 genes that were located within domestication related selective sweeps (Rubin et al. 2010).

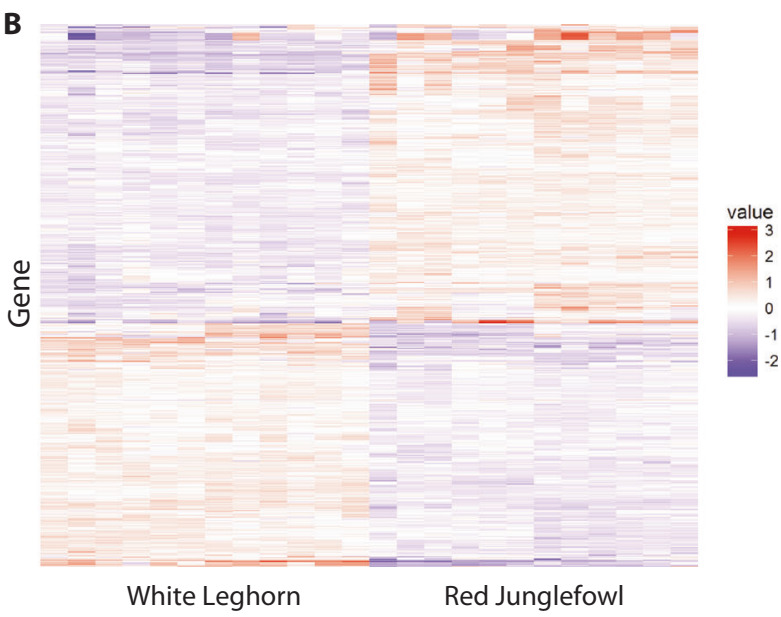

and log fold change $>0.5$ ). The first six samples (from left to right) for each breed are females and the rest are males. The full list of DE transcripts can be found in the Supplementary Table 3

\section{Results}

When comparing WL and RJF (gene expression $\sim$ breed + sex + treatment + breed $\times$ treatment), 489 annotated genes were found to be differentially expressed (DE) between the breeds (FDR corrected $p$-value threshold 0.05 and log fold change $>0.5$ ). Of these, 289 transcripts were found to be expressed at higher levels in RJF and 200 genes were expressed at higher levels in WL (Fig. 1 and Supplementary Table 3).

Out of the $489 \mathrm{DE}$ annotated genes, 430 were included in UniProt Knowledgebase (UniProtKB) database. GO analysis identified a significant enrichment (Benjamini corrected $p<0.01$ ) of genes in four functional categories, "Signaling", "Disulfide bond", "Glycoprotein", and "Secreted" (Fig. 2 and Supplementary Table 4). It is worth noting that an additional 412 transcripts from expressed sequence tags (dbEST) were found to be DE between breeds. DbEST is a collection of short single-read transcript sequences from GenBank (Boguski et al. 1993), that are not annotated and therefore were not included in any downstream analysis.

Next, we overlapped the breed DE list (489 genes) with a list of 407 specific genes. Based on differential tissue expression patterns, these genes have been suggested to have important functions in the pituitary gland of chickens at day 42 (Pritchett et al. 2017). Thirty-four genes were common to both lists, suggesting their importance in explaining the phenotypical and physiological differences between WL and RJF (Table 1). Overlapping breed DE genes with selective sweeps revealed 12 genes that were in both lists (Supplementary Table 3).

Subsequently, we selected 9 breed DE genes and 1 important pituitary gene (POMC) that was not amongst the 


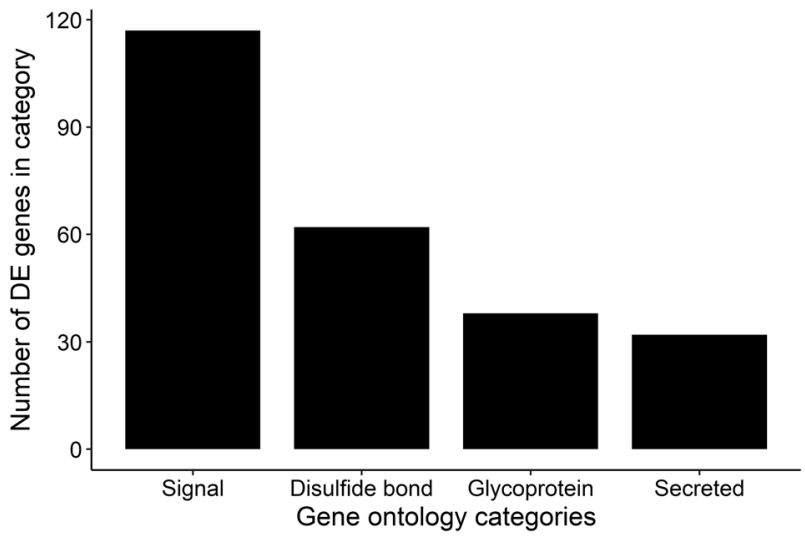

Fig. 2 Number of annotated genes differentially expressed between breeds that fell within each of the four significantly enriched gene ontology terms. Amongst the 430 genes with a known annotation, around 250 genes (including the genes which fell in more than one categories) fell within the significant categories. The uniport ID for Signal is KW-0732, Ddisulfide bond is KW-1015, glycoprotein is KW-0325 and for secreted is KW0964. The list of genes within each category is presented in Supplementary Table 4

DE gene list for validation with RT-qPCR. The expression patterns of 9 out of 10 genes agreed with the microarray results (Fig. 3 and Supplementary Table 5).

Comparing males and females, including breed and stress treatment in the model, 294 annotated genes were differently expressed, out of which, 37 were expressed higher in females, whereas 257 genes (165 Z-linked and 92 autosomal genes) were expressed higher in males. Overlapping sex DE gene list with the list of pituitary-important genes (407 genes) resulted in identification 23 genes that may play important sex specific role in chicken (Supplementary Table 6).

Stress treatment, affected expression of 6 genes, 3 of which were expressed higher after stress, whereas 3 were downregulated after stress Two of these stress DE genes also overlapped with the list of pituitary-important genes (Table 2 and Supplementary Table 7).

\section{Discussion}

We have used cDNA microarray to assess the effects of domestication and stress on the gene expression pattern in the pituitary gland of domesticated White Leghorn and Red Junglefowl. Around 500 annotated genes were differentially expressed between the breeds. Overlapping the breed DE list with the list of genes that are uniquely or more abundantly expressed in chicken pituitary gland (Pritchett et al. 2017) narrowed down the candidate gene list to 34 genes (Table 1). This candidate gene list contained genes that are known to affect central aspects of the domesticated phenotype, such as coat or plumage color, growth and sexual
Table 1 The list includes the subset of differently expressed genes between the domesticated White leghorn and Red Junglefowl based on the ANOVA model ( breed + sex + treatment), that overlaps with the list of pituitary-important genes at day 42 (Pritchett et al. 2017). Direction indicates whether the expression of the genes is higher in the domesticated White Leghorn or in the Red Junglefowl

\begin{tabular}{lll}
\hline Abbreviation & Gene name & Direction \\
\hline PRLR & Prolactin receptor & Higher in \\
KCNIP2 & Kv channel interacting protein 2 & WL \\
ENO4 & Enolase family member 4 & \\
OTOG & Otogelin & \\
S1X1 & SIX homeobox 1 & \\
ASIP & Agouti signaling protein
\end{tabular}

TCERG1L Transcription elongation regulator 1 like

ADAMTS18 ADAM metallopeptidase with thrombospondin type 1

KCNH7 Potassium voltage-gated channel, subfamily $\mathrm{H}$, member 7

PSD Pleckstrin and Sec7 domain containing

TMEM215 Transmembrane protein 215

LOC42098 HCG1657980

CCNA1 cyclin A1

TSHB Thyroid stimulating hormone beta

HESS Hes family bHLH transcription factor 5

FOXG1 Forkhead box G1

MMRN1 Multimerin 1

SNTN Sentan, cilia apical structure protein

AVPR1A Arginine vasopressin receptor 1A

FAM46D Family with sequence similarity 46member D

DIO2 Deiodinase, iodothyronine, type II

SSTR5 Somatostatin receptor 5

LOC428967 Gamma-aminobutyric acid receptor sub pi-like

COL4A4 Collagen, type IV, alpha 4

ZNF414 Zinc finger protein 414

Higher in

RJF

CDHR3 Cadherin related family member 3

TOM1L1 Targe tof myb1 like 1 membrane trafficking protein

\section{PENK Proenkephalin}

CLCA2 Chloride channel, calcium activated, member 2

CCDC39 Coiled-coil domain containing 39

SIX2 SIX homeobox 2

SOX3 SRY (sex determining region Y)-box 3

GPC3 Glypican 3

NR4A3 Nuclear receptor subfamily 4, group A, member 3 


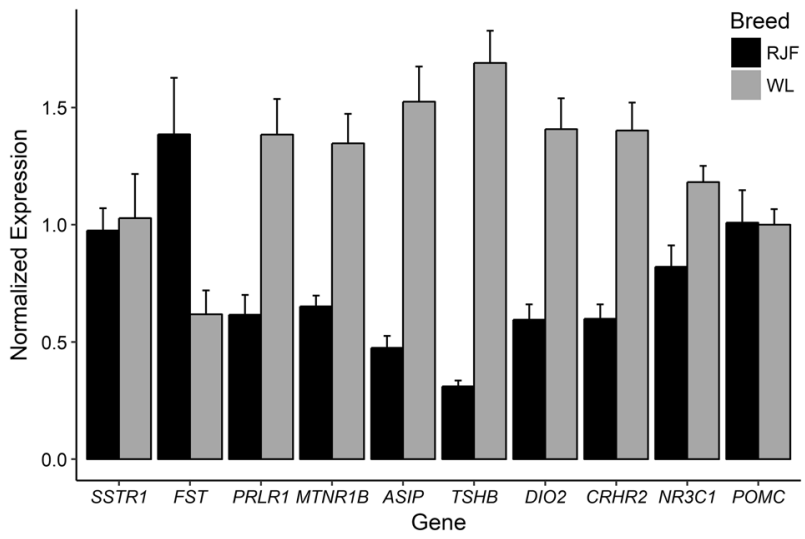

Fig. 3 Normalized relative expression (Delta $C_{\mathrm{t}}$ ) levels of genes showing differential expression between Red Junglefowl and White Leghorn, selected on grounds of overlaping with a list of pituitaryimportant genes or having known functions in processes relevant for the domesticated phenotype. Genes shown are somatostatin receptor type 1 (SSTR1), follistatin (FST), Prolactin receptor 1(PRLR1), melatonin receptor $1 B(M T N R 1 B)$, agouti signaling protein (ASIP), thyroid stimulating hormone beta (TSHB), deiodinase iodothyronine type II (DIO2), corticotropin releasing hormone receptor 2 (CRHR2), glucocorticoid receptor (NR3C1), and proopiomelanocortin (POMC). The used primers for RT_qPCR and results from statistical analysys can be found in Supplementary Table 2 and 5, respectively

Table 2 Annotated genes that were differentially expressed between control and stress treatment. The asterisk indicates that the gene is amongst the published pituitary-important genes (Pritchett et al. 2017). Direction indicates whether the gene is up or downregulated after $15 \mathrm{~min}$ of restraint stress

\begin{tabular}{lll}
\hline Abbreviation & Gene name & Direction \\
\hline PPP1R14D & $\begin{array}{l}\text { Protein phosphatase 1, regulatory } \\
\text { (inhibitor) subunit } 14 D\end{array}$ & $\begin{array}{l}\text { Higher after } \\
\text { stress }\end{array}$ \\
CYR61 & Cysteine rich angiogenic inducer 61 & $\begin{array}{l}\text { Higher after } \\
\text { stress }\end{array}$ \\
RGS1 & regulator of G-protein signaling 1 & $\begin{array}{l}\text { Higher after } \\
\text { stress }\end{array}$ \\
SLC1A3* & Solute carrier family member 3 & $\begin{array}{l}\text { Lower after } \\
\text { stress }\end{array}$ \\
G3BP2 & $\begin{array}{l}\text { Ras GTPase-activating protein- } \\
\text { binding protein 2 }\end{array}$ & $\begin{array}{l}\text { Lower after } \\
\text { stress }\end{array}$ \\
NDRG1* & N-myc downstream regulated 1 & Lower after \\
& & stress \\
\hline
\end{tabular}

Only one gene was found to show a significant breed $\mathrm{x}$ treatment interaction when correcting for multiple testing, the dual specificity protein phosphatase 4 (DUSP4) gene (adj. $p=0.04)$. In RJF, expression of DUSP4 increased after stress, whereas it decreased in WL

maturity, reproduction, and stress. The expression differences were in the direction expected based on the phenotypic differences between RJF and domesticated chickens.

Changes in body size and modification of coat or plumage color are generally associated with animal domestication (Price 1999). The gene coding agouti signaling peptide (ASIP), a key modulator of plumage or coat color was amongst the list of candidate genes (Table 1 and Fig. $3)$. Acting as an inverse agonist on melanocortin receptor 1 (MCR1), ASIP inhibits melanogenesis and promote yellow pigmentation (Norris and Carr 2013b). ASIP also promote feeding by blocking the appetite-suppressing effects of $\alpha$ MSH in the hypothalamus, and therefore, over-expression of this gene leads to obesity as well as dominance of yellow fur in mammals (Dreger and Schmutz 2011; Norris and Carr 2013a). Thus, it is likely that the observed higher expression of ASIP in WL is indeed associated with the well-known differences in color pattern and body size between domesticated WL and RJF.

Reaching sexual maturity at a younger age as well as a lack of strict seasonal reproduction are other shared characteristics in domesticated animals (Price 1999; Trut et al. 2009; Shinomiya et al. 2014). Thyroid stimulating hormone $\beta$ (TSHB) and deiodinase iodothyronine type II (DIO2) are localized in the pars tuberalis of the anterior pituitary and function as master regulators of puberty as well as seasonal reproduction in birds (Nakao et al. 2008; Karlsson et al. 2016; Nishiwaki-Ohkawa and Yoshimura 2016). Both $T S H B$ and $\mathrm{DIO} 2$ were expressed at higher levels in WL compared with RJF (Table 1 and Fig. 3), consistent with the earlier puberty and higher metabolic rate in the domesticated WL.

Prolactin receptor (PRLR) was amongst pituitaryimportant DE genes (Table 1) and was also amongst the top 10 breed DE genes (Figs 1a and 3). In birds, prolactin and PRLR are involved in regulation of maternal behavior and broodiness (Grommen et al. 2006; Dawson 2015). Prolactin secretion from the pituitary gland is controlled by the hypothalamus as well as by a negative feedback loop. This negative feedback is mediated through action of prolactin on PRLR at the level of the hypothalamus and pituitary gland (Chiu et al. 1992). Thus, higher expression of PRLR in the pituitary gland of WL may inhibit prolactin secretion and subsequently contribute to the lack of broodiness in this domesticated breed.

Besides the aforementioned genes that overlapped with the list of pituitary important genes, searching known stress modulatory genes revealed that $C R H R 2$ and $N R 3 C 1$, genes encoding hormone receptors involved in stress response, were also differentially expressed between breeds (Fig. 3). The glucocorticoid receptor (GR) is the main mediator of negative feedback of the HPA axis, and as we found a higher expression of the gene encoding GR, NR3C1, in WL. CRHR2 is also involved in modulation of the stress response. Investigations of mice deficient in CRHR2 have shown that these animals have higher levels of ACTH and corticosterone shortly after stress exposure (Preil et al. 2001). Thus, the higher expression of NR3C1 and CRHR2 we observe in WL may also be implicated in the reported 
dampened hormonal stress response in domesticated chickens (Soleimani et al. 2011; Ericsson et al. 2014).

Based on their biological roles in the regulation of seasonality and reproduction, we also selected FST and $M T N R 1 B$ for RT-QPCR validation. Expression levels of $F S T$ were higher in RJF, whereas, MTNRIB was expressed at higher levels in WL (Fig. 3). FST inhibits biosynthesis and secretion of FSH from the pituitary and thus inhibits puberty and reproduction (Herbison 2016). Lower gene expression levels of the inhibitory FST in the pituitary gland of the WL is consistent with higher egg laying capacity of this egg layer breed. Additionally, MTNRIB, which contributes to seasonally activated behavior such as molting (Chiu and Wise 1994; Wilkanowska et al. 2014), was expressed differently between the breeds, which may be related to the observed domestication effects on reproductive behavior.

To identify genomic regions associated with domestication, previous research has analyzed selective sweeps, i.e., chromosome regions with reduced genetic variation caused by selection of certain gene variants coupled with linkage (Rubin et al. 2010). Overlapping breed DE genes (489 genes) with annotated genes within selective sweeps (261 genes) showed that 12 genes were present in both list (Supplementary Table 3). Only one of these genes, nuclear receptor subfamily 4, group A, member 3 (NR4A3) was also present in the list of pituitary-important genes. In human, NR4A3 is suggested to play a key role in regulation of cell proliferation, differentiation, and metabolism (Pearen et al. 2006).

In the functional analysis, genes that were expressed differently were related to membrane components, posttranslational modification of secretory proteins, or secretion into the cell surroundings (Frand et al. 2000; Sevier and Kaiser 2002). Therefore, there was an overrepresentation of genes involved in different aspects of hormone secretion, suggesting that the activity of the pituitary gland differs in domesticated WL compared with RJF. However, the potential function of most genes that were DE between the breeds remains unknown. Domesticated chickens have diverged from the ancestral RJF at least 6000 years ago (Tixier-Boichard et al. 2011), and therefore, we can expect neutral fixation as well as divergent selection pressure to underlie the observed changes in the expression of hundreds of genes (Khaitovich et al. 2004).

Male and female birds usually differ extensively in a range of traits, including appearance and morphology, physiology and behavior. The hypothalamus and pituitary gland play key roles in modulation of physiological and morphological differences between the sexes (Scanes 2015). Around $165 \mathrm{Z}$-linked and 130 autosomal genes were differentially expressed between sexes. The fact that all DE Z-linked genes were expressed at a higher level in males is consistent with the well-known incomplete dosage compensation in chicken (Warnefors et al. 2017). Overlapping sex DE genes with the list of genes with important function in the pituitary gland revealed 23 genes which may contribute to development of sex specific characteristics in chickens (supplementary Table 6). Some of these genes such as gonadotropin-releasing hormone receptor (GNRHR), follicle stimulating hormone beta subunit (FSHB), and PRLR have a known function in regulation of reproduction, whereas the others present novel candidate genes for functional characterization. In the present study, the time from the onset of the stress treatment to sample collection was only $15 \mathrm{~min}$. We therefore expected the number of differently expressed transcripts to be low. Only 6 transcripts were found to differ between the treatments (Table 2 and Supplementary Table 7). Among the genes that were upregulated by the stress treatment were PPPIR14D and RSG1. PPPIRI4D encodes a protein that binds to and inhibits the activity of protein phosphatase 1 (PP1) (Liu et al. 2004; Eto 2009). PP1 is associated with both pre-mRNA slicing (Shi et al. 2006) and glycogen metabolism and regulation (Karthik and Ravikumar 2011), among other things. Inhibiting PP1 could impede slicing of RNA and thus play a role in gene regulation, but could also increase glycogen breakdown and reduce glycogen synthesis (Karthik and Ravikumar 2011). Currently, we do not know which of these roles the upregulation of PPP1R14D after stress has in the stress response.

$R S G 1$, also upregulated after restraint, encodes a protein that regulates $\mathrm{G}$ protein signaling. $R S G 1$ has also previously been shown to be upregulated in the pituitary of mice after $2 \mathrm{~h}$ of restraint stress treatment (Kim et al. 2010). Several G protein-coupled receptors are located in the pituitary, including AVPR1A, AVPR1B, CRHR1, CRHR2, MTNR1B, GnRH, and SSTR1 (Alexander et al. 2013), and it is possible that RGS1 may interact with any or several of these.

Only one gene, DUSP4 (also known as MAPK phosphatase, MKP-2), showed a significant interaction between breed and treatment. When the pituitary gland is stimulated by GnRH, MAPK pathways are activated, in turn increasing the expression of genes encoding subunits of the gonadotropins (Zhang and Roberson 2006). GnRH stimulation also increases the expression of DUSP4, mediated by early growth hormone 1 (EGR1) (Zhang et al. 2001). DUSP4 is a MAPK phosphatase that inactivates MAPK through dephosphorylation (Zhang et al. 2001; Zhang et al. 2001). Thus, the stimulation of DUSP4 expression by GnRH constitutes a negative feedback mechanism to desensitize the gonadotrophs to further stimulation by GnRH (Zhang et al. 2001; Zhang and Roberson 2006). This mechanism has been implicated in relaying GnRH pulse frequencies to the secretion rhythms of FSH and LH from gonadotrophs. 
Thus, the increase of DUSP4 after stress in the Red Junglefowl could indicate a desensitization of the gonadotrophs to stimulation by GnRH, whereas an opposite effect is observed in WL.

We have previously studied gene expression pattern in the hypothalamus of 21 days old and mature WL and RJF using microarrays (Nätt et al. 2012). Around 280 genes were DE between the breeds at both ages, however, we did not detect genes with known association with the domesticated phenotype in this list. Using RNA sequencing, (Albert et al. 2012) studied gene expression in brain frontal cortex in three pairs of mature domesticated and wild species (wolves and dogs, wild boars and pigs, and wild and domesticated rabbits). There was no overlap between the DE genes in the different domestication events (Albert et al. 2012).

Since previous studies of domestication effects on gene expression failed to show any consistent patterns in different parts of the brain (Saetre et al. 2004; Tymchuk et al. 2009; Albert et al. 2012; Nätt et al. 2012; Heyne et al. 2014), we suggest that domestication may primarily have acted on the pituitary. In that sense, the pituitary stands out as a potential central regulator of the domestication effect. We suggest that gene expression profiling of the hypothalamus and pituitary at different developmental stages in other populations of chickens as well as other domesticatedwild species pairs should be made to test whether our results are generalizable across species.

In conclusion, our results suggest that modified gene expression in the pituitary may account for phenotypic differences seen between domesticated chickens and ancestral RJF. We identified differential expression of genes involved in pigmentation, stress responses, growth, metabolism, and reproduction, and the direction of the observed differences were in line with the known phenotypic differences between the breeds. Our results therefore increase our current understanding of how biological pathways have been modulated by the domestication process.

\section{Data archiving}

The present experiment is uploaded on the Array Express website and is available under http://www.ebi.ac.uk/arra yexpress/experiments/E-MTAB-6269. Username: Reviewer_E-MTAB-6269, Password: nmmqJJKn

Acknowledgements The project was supported by grants from Swedish Research Council (VR): 2015-05444, the Swedish Research Council Formas: 2016-00645, and the European Research Council (ERC; Advanced Grant 322206 GENEWELL).

\section{Compliance with ethical standards}

Conflict of interest The authors declare that they have no conflict of interest.

\section{References}

Albert FW et al. (2012) A comparison of brain gene expression levels in domesticated and wild animals. PLoS Genetics. https://doi.org/ 10.1371/journal.pgen.1002962

Albert FW, Kruglyak L (2015) The role of regulatory variation in complex traits and disease. Nat Rev Genet 16(4):197-212. https:// doi.org/10.1038/nrg3891

Alexander SPH et al. (2013) The concise guide to pharmacology 2013/ 14: GPCRs. Br J Pharmacol 170:1459-1581. https://doi.org/10. 1111/bph.12444/full

Benjamini Y, Hochberg Y (1995) Controlling the false discovery rate: a practical and powerful approach to multiple testing. J R Stat Soc Ser. https://doi.org/10.2307/2346101

Boardman PE et al. (2002) A comprehensive collection of chicken cDNAs. Curr Biol 12(22):1965-1969. https://doi.org/10.1016/ S0960-9822(02)01296-4

Boguski MS, Lowe TMJ, Tolstoshev CM (1993) dbEST—database for "expressed sequence tags". Nat Genet 4(4):332-333. https:// doi.org/10.1038/ng0893-332

Chiu S, Koos RD, Wise PM (1992) Detection of prolactin receptor (PRL-R) mRNA in the rat hypothalamus and pituitary gland. Endocrinology 130(3):1747-1749. https://doi.org/10.1210/endo. 130.3.1537321.

Chiu S, Wise PM (1994) Prolactin receptor mRNA localization in the hypothalamus by in situ hybridization. J Neuroendocrinol 6 (2):191-199. https://doi.org/10.1111/j.1365-2826.1994.tb00572.x

Dawson A (2015) Avian Molting. (Chapter 38) In: Sturkie's Avian physiology, 6th edn, Academic Press, p 907-917. https://doi.org/ 10.1016/B978-0-12-407160-5.00038-5

Dreger DL, Schmutz SM (2011) A SINE insertion causes the blackand-tan and saddle tan phenotypes in domestic dogs. J Hered 102: S11-S18. https://doi.org/10.1093/jhered/esr042

Ericsson $\mathrm{M}$ et al. (2014) Domestication effects on behavioural and hormonal responses to acute stress in chickens. Physiol Behav 133:161-169. https://doi.org/10.1016/j.physbeh.2014.05.024

Eto M (2009) Regulation of cellular protein phosphatase-1 (PP1) by phosphorylation of the CPI-17 family, C-kinase-activated PP1 inhibitors. J Biol Chem 284(51):35273-35277. https://doi.org/10. 1074/jbc.R109.059972

Fallahsharoudi A et al. (2015) Domestication effects on stress induced steroid secretion and adrenal gene expression in chickens. Sci Rep 5:15345. https://doi.org/10.1038/srep15345.

Ferris HA (2006) Mechanisms for pulsatile regulation of the gonadotropin subunit genes by GNRH1. Biol Reprod 74(6):993-998. https://doi.org/10.1095/biolreprod.105.049049

Frand AR, Cuozzo JW, Kaiser CA (2000) Pathways for protein disulphide bond formation. Trends in cell biology 10(5):203-210

Grommen SVH et al. (2006) Molecular cloning, tissue distribution, and ontogenic thyroidal expression of the chicken thyrotropin receptor. Endocrinology 147(8):3943-3951. https://doi.org/10. 1210/en.2005-1223

Gulevich RG et al. (2004) Effect of selection for behavior on pituitaryadrenal axis and proopiomelanocortin gene expression in silver foxes (Vulpes vulpes). Physiol Behav 82(2-3):513-518. https:// doi.org/10.1016/j.physbeh.2004.04.062

Herbison AE (2016) Control of puberty onset and fertility by gonadotropin-releasing hormone neurons. Nat Rev Endocrinol 12 (8):452-466. https://doi.org/10.1038/nrendo.2016.70

Herman JP et al. (2016) Regulation of the hypothalamic-pituitaryadrenocortical stress response. Compr Physiol 6(2):603-621. https://doi.org/10.1002/cphy.c150015

Heyne HO et al. (2014) Genetic influences on brain gene expression in rats selected for tameness and aggression. Genetics 198 (3):1277-1290. https://doi.org/10.1534/genetics.114.168948 
Huang DW, Sherman BT, Lempicki RA (2008) Systematic and integrative analysis of large gene lists using DAVID bioinformatics resources. Nat Protoc 4(1):44-57. https://doi.org/10.1038/nprot. 2008.211

Jensen P (2014) Behavior genetics and the domestication of animals. Annu Rev Anim Biosci 2(1):85-104. https://doi.org/10.1146/a nnurev-animal-022513-114135

Joëls M, Baram TZ (2009) The neuro-symphony of stress. Nat Rev Neurosci 10(6):459-66. https://doi.org/10.1038/nrn2632

Johnsson $\mathrm{M}$ et al. (2016) Genetical genomics of behavior: a novel chicken genomic model for anxiety behavior. Genetics 202 (1):327-340. https://doi.org/10.1534/genetics.115.179010

Johnsson M, et al. (2018) Genetical genomics of growth in a chicken model BMC Genom 19(1):72. https://doi.org/10.1186/s12864018-4441-3

Karlsson A-CC et al. (2016) A domestication related mutation in the thyroid stimulating hormone receptor gene (TSHR) modulates photoperiodic response and reproduction in chickens. Gen Comp Endocrinol 228:69-78. https://doi.org/10.1016/j.ygcen.2016.02. 010

Karthik D, Ravikumar S (2011) Characterization of the brain proteome of rats with diabetes mellitus through two-dimensional electrophoresis and mass spectrometry. Brain Res 1371:171-179. https://doi.org/10.1016/j.brainres.2010.11.066

Keller-Wood M (2015) Hypothalamic-pituitary-adrenal axis-feedback control. Compr Physiol 5(3):1161-1182. https://doi.org/10.1002/ cphy.c140065

Kerje S et al. (2003) The twofold difference in adult size between the red junglefowl and White Leghorn chickens is largely explained by a limited number of QTLs'. Anim Genet 34(4):264-274. https://doi.org/10.1046/j.1365-2052.2003.01000.x

Khaitovich P et al. (2004) A neutral model of transcriptome evolution. PLoS Biol. https://doi.org/10.1371/journal.pbio.0020132

Kim G et al. (2010) Acute stress responsive RGS proteins in the mouse brain. Mol Cells 30(2):161-165. https://doi.org/10.1007/s10059010-0102-3

Künzl C, Sachser N (1999) The behavioral endocrinology of domestication: a comparison between the domestic guinea pig (Cavia aperea f. porcellus) and its wild ancestor, the cavy (Cavia aperea). Horm Behav 35(1):28-37. https://doi.org/10.1006/hbeh.1998. 1493

Liljedahl LE et al. (1979) Scandinavian selection and crossbreeding experiment with laying hens: i. background and general outline. Acta Agri Scand 29(3):273-286. https://doi.org/10.1080/ 00015127909435238

Liu Q et al. (2004) GBPI, a novel gastrointestinal- and brain-specific PP1-inhibitory protein, is activated by PKC and inactivated by PKA. Biochem J 377:171-181

Løtvedt P et al. (2017) Chicken domestication changes expression of stress-related genes in brain, pituitary and adrenals. Neurobiology of stress 7:113-121

Mirkena T et al. (2010) Genetics of adaptation in domestic farm animals: a review. Livest Sci 132(1-3):1-12. https://doi.org/10. 1016/j.livsci.2010.05.003

Nakao $\mathrm{N}$ et al. (2008) Thyrotrophin in the pars tuberalis triggers photoperiodic response. Nature 452(7185):317-322. https://doi. org/10.1038/nature06738

Nishiwaki-Ohkawa T, Yoshimura T (2016) Molecular basis for regulating seasonal reproduction in vertebrates. J Endocrinol. https:// doi.org/10.1530/JOE-16-0066

Norris DO, Carr JA (2013a) Chapter 12-chemical regulation of feeding, digestion and metabolism, vertebrate endocrinology, 5th edn. Elsevier. https://doi.org/10.1016/B978-0-12-394815-1.00012-4

Norris DO and Carr JA (2013b) Organization of the mammalian hypothalamus-pituitary axes, vertebrate endocrinology. Elsevier. https://doi.org/10.1016/B978-0-12-394815-1.00004-5.
Nätt D, et al. (2012) Heritable genome-wide variation of gene expression and promoter methylation between wild and domesticated chickens BMC Genom 13(1):59. https://doi.org/10.1186/ 1471-2164-13-59

Pearen MA et al. (2006) The orphan nuclear receptor, NOR-1, is a target of $\beta$-adrenergic signaling in skeletal muscle. Endocrinology 147(11):5217-5227

Plyusnina IZ, Solov'Eva MY, Oskina IN (2011) Effect of domestication on aggression in gray Norway rats. Behav Genet 41 (4):583-592. https://doi.org/10.1007/s10519-010-9429-y

Preil J et al. (2001) Regulation of the hypothalamic-pituitaryadrenocortical system in mice deficient for CRH receptors 1 and 2. Endocrinology 142(11):4946-4955

Price EO (1999) Behavioral development in animals undergoing domestication. Appl Anim Behav Sci 65(3):245-271. https://doi. org/10.1016/S0168-1591(99)00087-8

Pritchett EM et al. (2017) Transcriptomic changes throughout posthatch development in Gallus gallus pituitary. J Mol Endocrinol 58(1):43-55. https://doi.org/10.1530/JME-16-0186

Ritchie ME et al. (2015) limma powers differential expression analyses for RNA-sequencing and microarray studies. Nucleic Acids Res 43(7):e47-e47. https://doi.org/10.1093/nar/gkv007.

Rubin C-J et al. (2010) Whole-genome resequencing reveals loci under selection during chicken domestication. Nature 464 (7288):587-591. https://doi.org/10.1038/nature08832

Saetre P et al. (2004) From wild wolf to domestic dog: gene expression changes in the brain. Mol Brain Res 126(2):198-206. https://doi. org/10.1016/j.molbrainres.2004.05.003.

Scanes CG (2015) Pituitary Gland. (Chapter 23) In: Sturkie's Avian Physiology. Academic Press, p 497-533. https://doi.org/10.1016/ B978-0-12-407160-5.00023-3.

Sevier CS, Kaiser CA (2002) Formation and transfer of disulphide bonds in living cells. Nature reviews Molecular cell biology 3 (11):836

Schütz K et al. (2002) 'QTL analysis of a red junglefowl $\times$ White Leghorn intercross reveals trade-off in resource allocation between behavior and production traits'. Behav Genet 32 (6):423-433. https://doi.org/10.1023/A:1020880211144

Schütz KE, Forkman B, Jensen P (2001) Domestication effects on foraging strategy, social behaviour and different fear responses: A comparison between the red junglefowl (Gallus gallus) and a modern layer strain. Appl Anim Behav Sci 74(1):1-14. https:// doi.org/10.1016/S0168-1591(01)00156-3

Shi Y, Reddy B, Manley JL (2006) PP1/PP2A phosphatases are required for the second step of pre-mRNA splicing and target specific snRNP proteins. Mol Cell 23(6):819-829. https://doi.org/ 10.1016/j.molcel.2006.07.022

Shinomiya A et al. (2014) Regulation of seasonal reproduction by hypothalamic activation of thyroid hormone. Front Endocrinol. https://doi.org/10.3389/fendo.2014.00012.

Soleimani AF et al. (2011) Physiological responses of 3 chicken breeds to acute heat stress. Poult Sci 90(7):1435-1440. https:// doi.org/10.3382/ps.2011-01381

Tixier-Boichard M, Bed'Hom B, Rognon X (2011) Chicken domestication: from archeology to genomics. Comptes Rendus - Biologies. https://doi.org/10.1016/j.crvi.2010.12.012

Trut L, Oskina I, Kharlamova A (2009) Animal evolution during domestication: the domesticated fox as a model. Bioessays 31 (3):349-360. https://doi.org/10.1002/bies.200800070.Animal

Tymchuk W, Sakhrani D, Devlin R (2009) Domestication causes large-scale effects on gene expression in rainbow trout: Analysis of muscle, liver and brain transcriptomes. Gen Comp Endocrinol 164(2-3):175-183. https://doi.org/10.1016/j.ygcen.2009.05.015

Warnefors $M$ et al. (2017) Sex-biased microRNA expression in mammals and birds reveals underlying regulatory mechanisms 
and a role in dosage compensation. Genome Res. https://doi.org/ 10.1101/gr.225391.117.Freely

Weiler $U$ et al. (1998) Influence of age and genotype on endocrine parameters and growth performance: a comparative study in Wild boars, Meishan and Large White boars. Livest Prod Sci 54 (1):21-31. https://doi.org/10.1016/S0301-6226(97)00165-6

Wickham H (2009) ggplot2 Elegant graphics for data analysis. Media. https://doi.org/10.1007/978-0-387-98141-3.

Wiener P, Wilkinson S (2011) Deciphering the genetic basis of animal domestication. Proc Biol Sci 278(1722):3161-3170. https://doi. org/10.1098/rspb.2011.1376

Wilkanowska A et al. (2014) Prolactin (PRL) and prolactin receptor (PRLR) genes and their role in poultry production traits. Folia Biol. https://doi.org/10.3409/fb62_1.1
Yates A et al. (2016) Ensembl 2016. Nucleic Acids Res 44(D1): D710-D716. https://doi.org/10.1093/nar/gkv1157

Zhang T, Mulvaney JM, Roberson MS (2001) Activation of mitogenactivated protein kinase phosphatase 2 by gonadotropin-releasing hormone. Mol Cell Endocrinol 172(1-2):79-89

Zhang T, Roberson MS (2006) Role of MAP kinase phosphatases in GnRH-dependent activation of MAP kinases. J Mol Endocrinol 36(1):41-50. https://doi.org/10.1677/jme.1.01881

Zhang T, Wolfe MW, Roberson MS (2001) An early growth response protein (Egr) 1 cis-element is required for gonadotropin-releasing hormone-induced mitogen-activated protein kinase phosphatase 2 gene expression. J Biol Chem 276(49):45604-45613. https://doi. org/10.1074/jbc.M107075200 